\title{
Effects of different protocols of strength training in postoperative rehabilitation of anterior cruciate ligament: a review
}

\begin{abstract}
Rehabilitation protocols following anterior cruciate ligament repair surgery are being subject to multiple changes and studies. In order to minimize chronic muscle weakness and atrophy, strength training is being used even in the earlier stages after surgery. Scientific studies seem to support the use of strength exercises both in open and closed kinetic chain starting at early stages after surgery. Has also been demonstrated the utility and safety of strength exercises in regime of eccentric contraction.
\end{abstract}

Keywords: anterior cruciate ligament, knee joint, quadriceps muscle, strength training
Volume 2 Issue 2 - 2018

\author{
Andrés Parodi Feye \\ Instituto Superior de Educación Física, Universidad de la \\ República, Uruguay
}

\begin{abstract}
Correspondence: Andrés Parodi Feye, Instituto Superior de Educación Física, Universidad de la República, Uruguay, Tel 0598 973। 4497, Email andresparodi2005@yahoo.com
\end{abstract}

Received: March 30, 2018 | Published: April 19, 2018

\section{Introduction}

Soft tissue knee surgery is performed on multiple conditions and comprises a large number of procedures. The postoperative management of these patients has been subject of multiple reviews and has been subject to constant changes. While it has been proposed that the rehabilitation process is as important as surgery and the large number of scientific studies focused on this issue, there is no consensus among authors above the protocols most suitable to follow for each case, ${ }^{1}$ although it is well established that exercise therapy constitutes a substantial part of a rehabilitation program. ${ }^{2}$ In subjects undergoing an anterior cruciate ligament (ACL) surgery, an appropriate and carefully designed post-operative rehabilitation strategy is necessary to allow the return to functional activities of daily life and sport activity safely and effectively, preventing the premature development of knee osteoarthritis. ${ }^{3}$ This rehabilitation program usually begins in the acute stage after surgery (including the same day) and includes a variety of treatment modalities, as cryotherapy, gait re-education, joint mobility exercises, continuous passive mobility of the joints involved, strength exercises, electrical stimulation and compressive bandages. ${ }^{4,5}$

Atrophy and the concomitant loss of muscle strength of involved lower limb, particularly of the quadriceps femoris muscle (this being the most important muscle for the function of the mentioned limb) are predominant disabilities after such intervention. The extent of the atrophy and loss of strength often exceed $20 \%$ and $30 \%$ respectively, during the first three months ${ }^{6}$ and the deficit may persist, in a magnitude of $10 \%$ to $20 \%$, for years after the surgery, even after the application of post-operative rehabilitation program. Such weakness, accompanying the ACL injury, is linked to loss of functional performance, increasing in the risk of re-injury and the development of post-traumatic osteoarthritis. ${ }^{8,9}$ An important factor contributing to post-traumatic persistent weakness of the quadriceps femoris is the arthrogenic muscle inhibition, which implies a reflex mechanism of response to injury joint. Although this inhibition probably represents a mechanism of defense prior to the injury, may become a limiting factor to the postoperative rehabilitation purposes. ${ }^{10}$ This loss of strength of the affected limb may persist even many years after surgery, as evidenced by the study of Tengman et al. ${ }^{11}$ who evidenced in both sexes, more than 20 years after the operation, a significant difference in the maximum force extension of knee, both in concentric as eccentric contractions, between the operated leg and the contralateral one, being the first at least a $10 \%$ weaker than the second. There was also a loss of strength of the affected leg, during maximum flexion of knee in eccentric contraction. ${ }^{11}$

In this regard, it is necessary to create protocols involving feasible, safe and effective muscle strength training at early stage after surgery, in order to minimize chronic muscle weakness and atrophy. Our problem in the selection of exercises for postoperative rehabilitation is the limited knowledge about the optimum timing to adjust training loads and the choice of exercises and its intensity, in order to avoid overloading and consequently cause injury, in the graft. Several studies have shown that load that suffers the ACL graft is generally greater in exercises that involve the own body weight that in periods with weight-bearing exercises: in both cases, the load on the mentioned graft is higher between $10^{\circ}$ and $50^{\circ}$ of knee flexion (and particularly, between $10^{\circ}$ and $30^{\circ}$ ), compared with $50^{\circ}$ to $100^{\circ}$ of flexion. ${ }^{12}$

One of the main objectives of ACL rehabilitation is to restore the strength of the muscles on the operated joint ${ }^{13}$ and quadriceps femoris muscle strengthening seems to play a main role. ${ }^{14}$ This muscle, as well as being the main knee extensor, has been pointed out as the main dynamic stabilizer of the joint during closed kinetic chain activities, which are those that predominate in everyday life and in sport. ${ }^{15}$ It has also been suggested that both the pain and the weakness of the quadriceps femoris are important predictors of disability, after ACL reconstruction. ${ }^{16}$ Rehabilitation protocols used in the 1990s, usually involved the operated knee immobilization for at least six weeks, employing after that resisted knee extension exercises, primarily in open kinetic chain. ${ }^{17-19}$ The use of weight-bearing excersies for muscles involving the knee joint was often delayed several weeks to prevent ligament laxity and protect the graft through immobilization. ${ }^{12}$

Even though they were designated to protect from excessive stress on the extensor mechanism of the joint ${ }^{20}$ this type of protocols came into conflict with the knowledge, already established, of the damaging effect of the deficient stimulation, in terms of strength, of the stabilizing muscles if the knee joint. ${ }^{3}$ In subsequent years, protocols started to include the use of different regimes of strength exercises, during the early stage of rehabilitation. However, the use of a very aggressive exercise strategy prematurely can cause excessive stress 
on the graft implantation site and still not generate the desired torque in the extension of knee. ${ }^{21}$ In the majority of cases, the affected tissues heal in response to a load of adequate strength intensity; however, the deformation forces are harmful and therefore should be avoided that ACL graft receives this type of loads that may eventually lead to its rupture. ${ }^{22}$

In this regard, Margit et al. ${ }^{12}$ carried out a study in order to assess the effectiveness of the immediate use of overload (weight-bearing) exercises as compared to the use of this type of exercises starting from the second week of LCA post-operation. In this paper, 30 patients were assigned to one of two groups: the first group, used overload exercises in open kinetic chain (OKC) and in closed kinetic chain (CKC) from the first day after surgery, according to tolerance of the patients. By contrast, subjects in the second group conducted overload exercises just after the second week after surgery and exclusively in OKC. Regardless of strength training, in both groups a rehabilitation program began with other therapeutic measures from the first day after surgery. After 6 months after operation, the authors found no significant differences between both groups, but it was evident an improvement in the stability of the knee when compared to the rehabilitation assessment in both groups. The authors recommend in their conclusions the use of both "free" and overloaded exercises and both $\mathrm{OKC}$ and $\mathrm{CKC}$ exercises, considering that all of them are effective and safe in the postoperative recovery of the ACL reconstruction.

It is considered that an exercise is performed in CKC when the distal area of the limb makes the effort (in this case, lower limb) is fixed to a surface as the ground and therefore it cannot move freely. This type of exercise typically involve squats, lunges and leg press and involve the co-contraction of the muscles surrounding the joint involved (in this case, quadriceps femoris, hamstrings, hip flexors and muscles of the calf). On the other hand, an exercise in OKC involves that the distal area of the Member is free to move and they typically include extensions of knee, straight leg elevations, hamstring curls and back kicks. ${ }^{2}$ Many authors recommend the use of strength exercises in closed kinetic chain (CKC), arguing that these type of exercise helps to restore proprioceptive capacity and the stability of the knee, implying less tension for the graft as well as being better tolerated for the patient compared with open kinetic chain (OKC) exercises. Exercises in CKC are then more functional and safer compared to exercises in OKC. ${ }^{23}$ In addition, Kvist \& Gillquist ${ }^{24}$ posits that exercises in CKC produce smaller anterior translation of the tibia than exercises in OKC, while other authors have reported anterior translation of the tibia and similar shear forces on the graft in exercises at $\mathrm{CKC}$ compared with exercises at $\mathrm{OKC} .{ }^{25}$

The advantages of the use of exercises at CKC include reduction of shear and forces of acceleration on the knee joint, early development of dynamic stability and stimulation of the proprioception capacity. ${ }^{1}$ In this regard, Kruse et al. posits that, based upon their bibliography review, the use of overload and strengthening exercises in CKC immediately after surgery appear to be safe, while the employment of strength exercises in eccentric contraction and Isokinetic regimes starting from the third week after surgery could improve or accelerate the strength gains. However, patients treated exclusively with CKC exercises have shown difficulty in gaining sufficient muscular torque to return to his sport after 6 months, compared to the combined use of exercises in both kinetic chains.

It has been reported that increases in load of the quadriceps femoris during OPK exercises generated an increase of the maximum stress on the ACL, whereas this does not happens with a similar increase in load during an exercise in closed kinetic chain. ${ }^{26}$ For this reason and in order to prevent an excessive strain on the ACL, many current rehabilitation protocols use the latter instead of the former to strengthen the quadriceps femoris muscle, which seems in turn to play a primary role in the rehabilitation of ACL. ${ }^{14}$

There are concerns that the OKC exercise can cause shear forces in the femoro-patellar joint, therefore damaging the correct implementation of the graft. However, the scientific evidence in this respect is not conclusive, having found no significant differences in gait analysis (both walking on flat as well as up and down stairs), in anterior soreness of the knee or laxity of the joint when rehabilitation protocols involving OKC exercises were compared or CKC. However, some studies have shown significant increases in torque of quadriceps femoris with the combined use of both kinetic chain exercises and a significantly higher proportion of patients could return to their sport activities earlier. For this reason, the scientific literature seems to favor the combination of exercises of $\mathrm{CKC}$ and $\mathrm{OKC}$ for a maximum training effect. ${ }^{2}$

In accordance with this, Mikkelsen et al. ${ }^{27}$ found that the use of quadriceps femoris strengthening exercises in both open kinetic chain and close kinetic chain, starting from the sixth week after surgery, was superior to use of exercises in closed kinetic chain only, in terms of an improvement of muscle torque and a significantly faster return to sport activity. Other types of strength exercises that have been used and studied as part of a rehabilitation protocol involve the use of exercises with an isometric contraction regime. In this regard, Shaw et al. ${ }^{28}$ studied the effect of the strengthening of the quadriceps femoris muscle early after surgery, by randomly assigning 103 patients to one of two groups: a group of "quadriceps femoris strengthening" and a group of "not quadriceps femoris exercise". The main difference between them was that the former was instructed to perform static quadriceps exercises, as well as elevations of "Straight-Leg-Raise" on a daily basis (10 repetitions, three times per day) during the first two weeks after surgery, while the latter did not at this stage any exercise of strengthening. After those first two weeks, all patients continued with the same standard program of rehabilitation.

During six months of follow-up, these authors found no significant differences, resulting from the use during the acute stage after surgery of quadriceps femoris strength exercises, when it comes to muscle strength or functionality of the lower limb. However, they mentioned that subjects who exercised their quadriceps had a faster recovery in terms of range of motion and best score in the self-administered questionnaire Cincinnati Knee Rating System. The capacity of a muscle to produce force and therefore his response both structural and functional, is greater when an external force overcomes the force of that muscle, making it contract eccentrically. ${ }^{29}$ It has been shown that the application, incremental and progressively, of eccentric strength exercises, increases the size and muscle strength in healthy population as well as in patients with osteo-articular pathology safely and can consequently be applied as part of a protocol of rehabilitation after ACL reconstruction. Important gains in size and strength of the quadriceps femoris as a result of eccentric training on CKC after reconstruction of the ligament were verified in a case study report conducted by Gerber et al. ${ }^{30}$ The same authors demonstrated that the gradual and progressive implementation of eccentric strength training can be tolerated without deleterious side effects after such surgery. ${ }^{31}$

In another study, ${ }^{31} 40$ adults (aged 18-50) moderately active (scores in the scale of activity of Tegnér $\geq 4$ ) that had rupture and subsequent surgical reconstruction of ACL, were randomly assigned 
to two groups: standard rehabilitation group and rehabilitation with eccentric training group. Both groups followed a rehabilitation program considered of reference of three phases, which puts emphasis on closed chain, functional training exercises and in gain early range of motion of the knee joint. Phase I lasted 2-3 weeks and consisted of exercises focused on controlling the pain and swelling, achieving a range of motion of the joint knee at least 0 to $100^{\circ}$ and the repurchasing of the basic functions of the quadriceps femoris muscle. In phase II, both groups continued for 12 weeks with standard rehabilitation, with the difference of that eccentric training group was instructed to carry out progressively from 5 to 30 minutes of eccentric cycloergometer, gradually progressing to intensity perceived as hard on the scale of subjective perception of Borg. In order to match the volume of training of both groups, the standard training group followed a similar exercise regimen, but using a concentric cycloergometer. Phase III consisted, in both groups, of strength and endurance training exercises, jogging and running progression, plyometric exercise and preparation for competitive sport.

Completed the rehabilitation protocol, there was an increase in volume and maximum cross sectional area in quadriceps and distal portion of the gluteus maximus of both lower limbs (measured by Nuclear Magnetic Resonance, NMR) in both groups. However, this increase was more than $100 \%$ higher in the group of eccentric training than in the standard training group, something that was verified in both semitendinosus and patellar tendon graft patients. The authors concluded, since forces produced in a muscle during a negative work are of a greater magnitude that any contraction, an intervention with eccentric exercise may be ideal to mitigate persistent deterioration muscle commonly observed after ACL reconstruction. Mentioned improvements in muscle volume, as well as the profits of maximum torque of muscle quadriceps femoris and the unipodal jump test verified in both groups, but to a greater extent in the eccentric training group, remained even after a year following surgery, as it was clear in a follow-up study carried out for the same authors. The effect of early strengthening of hamstrings muscles after ACL reconstruction was also studied. Sekir et al..$^{32}$ studied the isokinetic strengthening in this muscle group in patients who had undergone ACL reconstruction using an autograft with patellar tendon. In this study, 48 patients were randomly assigned to one of two groups: a group began a program of isokinetic hamstrings exercise starting three weeks after surgery, while the other group did the same but starting at nine weeks after it.

Patients were then evaluated monthly for a period of four months and were then evaluated one year post-operation using for that purpose the subjective questionnaire of knee of the International Knee Documentation Committee (IKDC score), the knee score of Cincinnati and evaluating the hamstrings isometric and isokinetic force. The results showed that the early strengthening (three weeks after surgery) was associated with an increase in isometric and isokinetic force (measured at $60^{\circ}$ per second) of this muscle group and in addition this group shows significant improvements in everyday life activities, although there was not differences in the IKDC score between both groups. It is relevant to note that the early strengthening of hamstrings was not associated with deleterious effects on the joint surgically intervened. Another group of researchers investigated the effect of early and progressive strength training; both in concentric and eccentric regime, in functional performance of the knee joint after autologous reconstruction of ACL. ${ }^{33}$ For this purpose, 33 patients with autologous ligamentoplasty from hamstring muscles were randomly assigned to one of two groups: experimental group and control group.
Both groups followed the same standard protocol of postoperative rehabilitation, but in addition, the former follows a 12 weeks eccentric and concentric muscle training program at $\mathrm{CKC}$, starting three weeks after surgery and using a specially designed fitness device (Monitorized Functional Squat System) for this purpose.

The authors found, after 16 weeks post-surgery, a significant improvement in the experimental group compared with the control group, with regard to functional performance (evaluated with unipodal vertical and horizontal jump test), the scale of Lysholm (used to classify the patients' subjective feeling of satisfaction with their acquired functional ability) and the ACL-QOL post-surgery quality of life questionnaire. There were not, however, statistically significant differences between the two groups in regards to isokinetic force of flexor and extensor of the knee joint. Other clinical modality used to increase strength and muscle mass in patients undergoing treatment of ACL ligamentoplasty is the Electrical Neuromuscular Stimulation (ENMS), which has the capacity to initiate action potentials in intramuscular nerve roots, thus causing involuntary contractions of the stimulated muscle. In a study ${ }^{34} 36$ subjects undergoing surgery of ACL ligamentoplasty were assigned to one of four treatment groups: all participants conducted a standard treatment of rehabilitation, but additionally the Group I made ENMS and eccentric muscle training, group II made only eccentric muscle training, group III made only ENMS and Group IV performed only the standard treatment. Ten healthy subjects participated in the study as a control group.

Group I and III received ENMS treatment twice per week (10 isometric contractions for 10 seconds per session), during the first six weeks post-reconstruction, while the Group I and II did two eccentric muscle training twice a week, but starting at the sixth week post-reconstruction, on a leg press specially designed for this purpose (BLAST! ${ }^{\mathrm{TM}}$ Leg Press), doing 10 isokinetic eccentric contractions of quadriceps femoris muscle with the operated leg, totaling $4 \times 2$ minutes of recovery. In this prospective study was verified that therapeutic intervention involving the combined use of ENMS and eccentric muscle training or eccentric training only (groups I and II) recovered quadriceps femoris force greater than patients who only received ENMS or who only did the "standard" treatment (groups III and IV) after the ACL reconstruction. In groups I and II, femoral quadriceps activation levels reached similar levels to healthy subjects in the control group.

The authors concluded that eccentric exercise therapy, but not the ENMS, was the key factor behind the improvements in postACL ligamentoplasty quadriceps femoris activation; in this sense, they suggest that eccentric exercise represent an effective therapy, making improvements in force that match or even exceed the levels that patients had prior to the injury. These conclusions are consistent with that found by Brazilian et al. ${ }^{35}$ who studied the morphological and functional recovery of quadriceps femoris muscle in patients undergoing ACL reconstruction, after rehabilitation that involved eccentric muscle training protocol. In this study, 9 adult sedentary males (average 31 years), that had been unilateral ACL reconstruction using patellar tendon plasty since 8 to 9 months (and therefore were in the late phase of rehabilitation) were subjected to maximum eccentric training sessions only for the lower limb treated, using a isokinetic dynamometer, at a rate of $30^{\circ}$ per second, with a frequency of twice per week for 12 weeks.

Once completed the training protocol, significant improvements in torque of quadriceps femoris of lower limb intervened were evidenced, 
although still persisted a residual deficit between $18 \%$ and $29 \%$, in comparison with the contralateral lower limb. The authors of this study conclude that, in the first instance, increase in knee extension torque is determined, in by a combination between morphological and neural factor, while trophic factors are predominant in later stages of the protocol of intervention. They also mention that eccentric muscle training is a powerful therapeutic resource for both increasing in muscle strength and tropic recovery.

\section{Conclusion}

The scientific literature review presented in this paper allow us to establish that there is a consensus among authors about the importance of strength training as part of a protocol of rehabilitation after ACL reconstruction. It has also been established that strength training during an early stage after surgery, both in isometric and concentric regimen, is safe and even beneficial in that process. Eccentric strength training, applied in later stages of rehabilitation (at least after three weeks post-surgery) have proven to be much more effective that other regimens of force, with respect to strength gains in their different manifestations and in regards to muscle hypertrophy, particularly of quadriceps femoris muscle. It remains to establish whether the use of eccentric force from the very early stages of rehabilitation, or even prior to surgical intervention, is a safe and effective strategy, in regards to the functional rehabilitation of the patient

\section{Acknowledgements}

None.

\section{Conflict of interests}

Author declares that there is no conflict of interest.

\section{References}

1. Saka T. Principles of postoperative anterior cruciate ligament rehabilitation. World J Orthop. 2014;5(4):450-459.

2. Dushan H, Atkinson E, Laver J, et al. Physiotherapy and rehabilitation following anterior cruciate ligament reconstruction. Orthopaedic World Literature Society, 2009.

3. Grodski M. Exercises following anterior cruciate ligament reconstructive surgery: biomechanical consideratons and efficaciy of current approaches. Res Sports Med. 2008;16(2):75-96.

4. Chipchase L, Brumby S. In-Patient Physiotherapy Management of Orthopaedic Surgery. Oxford: Butterworth-Heinemann; 2001.

5. Carlo DM, Klootwyk T, Oneacre K. Anterior Cruciate Ligament. In: T Ellenbecker, editor. Knee Ligament Rehabilitation, Philadelphia; 2000. p. $117-129$

6. Feller J, Webster KE. A randomized comparision of patellar tendon and hamstring tendon anterior cruciate ligament reconstruction. Am J Sports Med. 2003;31(4):564-573.

7. Mattacola CG, Perrin DH, Gansneder BM. STrenght, functional outcome, and postural stability after anterior cruciate ligament reconstruction. $J$ Athl Train. 2002;37(3):262-268.

8. Tourville TW, Jarrell KM, Naud S, et al. Relationship Between Isokinetic Strength and Tibiofemoral Joint Space Width Changes After Anterior Cruciate Ligament Reconstruction. Am J Sports Med. 2014;42(2):302-311.

9. Schmitt LC, Paterno MV, Hewett TE. The Impact of Quadriceps Femoris Strength Asymmetry on Functional Performance at Return to Sport
Following Anterior Cruciate Ligament Reconstruction. J Orthop Sports Phys Ther. 2012;42(9):750-759.

10. Hart JM, Pietrosimone B, Hertel J, et al. Quadriceps activaton following knee injuries: a systematic review. J Athl Train. 2010;45(1):87-97.

11. Tengman E, Olofsson BL, Stensdotter AK, et al. Anterior cruciate ligament injury after more than 20 years. II. Concentric and eccentric knee muscle strength. Scand J Med Sci Sports. 2014;24(6):501-509.

12. Hidi M, Gergely I, Tudor S, et al. Comparative Study on the Effectiveness of Early or Delayed Weight-Bearing After Anterior Cruciate Ligament Reconstruction. Acta Médica Marisiensis. 2016;234-238.

13. Pfeifer K, Banzer W. Motor performance in different dynamic tests in knee rehabilitation. Scand J Med Sci Sports. 1999;9(1):19-27.

14. Bynum EB, Barrack RL, Alexander AH. Open versus closed chain kinetic exercises after anterior cruciate ligament reconstruction. A prospective randomized study. Am J Sports Med. 1995;23(4):401-406.

15. Bodor M. Quadriceps protects the anterior cruciate ligament. J Orthop Res. 2001;19(4):629-633.

16. Risberg MA, Holm I, Tjomsland O. Prospective studies on changes in impairments and disabilities after anterior cruciate ligament reconstruction. J Orthop Sports Phys Ther. 1999;29(7):1337-1345.

17. Stanish WD, Lai A. New concepts of rehabilitation following anterior cruciate reconstruction. Clin Sports Med. 1993;12(1):25-58.

18. Lieber R. Skeletal muscle structure and function. London: Williams \& Wilkins; 1992.

19. Frndak PA, Berasi CC. Rehabilitation concerns following anterior cruciate ligament recontruction. Sports Med. 1991;12(5):338-346.

20. Morrissey MC, Drechsler WI, Morrissey D. Effects of distally fixated versus nondistally fixated leg extensor resistance training on knee pain in the early period after anterior cruciate ligament reconstruction. Phys Ther. 2002;82(1):35-43.

21. Heijne A, Werner S. Early versus late start of open kinetic chain quadriceps exercises after ACL reconstruction with patellar tendon or hamstring graftws: A prospective randomized outcome study. Knee Surgery Sports Traumatology. 2007;15(4):402-414.

22. Hen K, On C, Laszlo I. Rehabilitation programs after anterior cruciate ligament reconstruction in highly active individuals: a review. Palestrica Mileniului III - Civilizatie si Sport. 2011;159-163.

23. Fitzgerland G. Open versus closed kinetic chain exercise: issues in rehabilitation after anterior cruciate ligament reconstructive surgery. Phys Ther. 1997;1747-1754.

24. Kvist J, Gillquist J. Sagittal plane knee translation and electromyographic activity during closed and open kinetic chain exercises in anterior cruciate ligament-deficient patients and control subjects. Am J Sports Med. 2001;29(1):72-82.

25. Isaac DL, Beard DJ, Price AJ, et al. In-vivo sagittal plane knee kinematics: ACL intact, deficient and reconstructed knees. Knee. 2005;12(1):25-31.

26. Fleming BC, Ohlén G, Renström PA, et al. The effects of compressive load and knee joint torque on peak anterior cruciate ligament strains. $\mathrm{Am}$ J Sports Med. 2003;31(5):701-707.

27. Mikkelsen C, Werner S, Eriksson E. Closed kinetic chain alone compared to combined open and closed kinetic chain exercises for quadriceps strenghtening after anterior cruciate ligament reconstruction with respects to return to sports: a prospective matched follow up study. Knee Surg Sports Traumatol Arthrosc. 2000;8(6):337-342.

28. Shaw T, Williams MT, Chipchase LS. Do early quadriceps exercises 
affect the outcome of ACL reconstruction? A randomised controlled trial. Aust J Physiother. 2005;51(1):9-17.

29. Lindstedt SL, LaStayo PC, Reich TE. When active muscles lenghten: properties and consequences of eccentric contractions. News Physiol Sci. $2001 ; 16: 256-261$.

30. Gerber JP, Marcus RL, Dibble LE. Early application of negative work via eccentric ergometry following anterior cruciate ligament reconstruction: a case report. J Orthop Sports Phys Ther. 2006;36(5):298-307.

31. Gerber JP, Marcus RL, Dibble LE. Safety, feasibility and efficacy of negative work exercise via eccentric muscle activity following anterior cruciate ligament reconstruction. J Orthop Sports Phys Ther. 2007;37(1):10-18.

32. Sekir U, Gur H, Akova B. Early versus late start of isokinetic hamstring- strengthening exercise after anterior ligament reconstruction with patellar tendon graft. Am J Sports Med. 2010;38(3):492-500.

33. Kınıklı GI, Yüksel I, Baltacı G, et al. The effect of progressive eccentric and concentric training on functional performance after autogenous hamstring anterior cruciate ligament reconstruction: a randomized controlled study. Acta Orthop Traumatol Turc. 2014;48(3):283-289.

34. Lepley LK, Wojtys EM, Smith PRM. Combination of Eccentric Exercise and Neuromuscular Electrical Stimulation to Improve Quadriceps Function Post-ACL Reconstruction. Knee. 2015;22(3):270-277.

35. Brasileiro JS, Pinto OM, Avila MA, et al. Functional and morphological changes in the quadriceps muscle induced by eccentric training after ACL reconstruction. Rev Bras Fisioter. 2011;15(4):284-290. 